\title{
Multivariable Nonlinear Predictive Control of Cement Mills
}

\author{
Lalo Magni, Georges Bastin, and Vincent Wertz
}

\begin{abstract}
A new multivariable controller for cement milling circuits is presented, which is based on a nonlinear model of the circuit and on a nonlinear predictive control strategy. Comparisons with previous $L Q$ control strategies show improved performances with respect to an important source of perturbations of the circuit: a change of hardness of the raw material.
\end{abstract}

Index Terms-Cement industry, multivariable control systems, nonlinear systems, optimal control, predictive control.

\section{InTRoduction AND Process Description}

$\mathbf{T}$ THE introduction of modern control methodologies in traditional process industries is in steady growth. Modelbased controllers usually allow to tackle multivariable control where classical PID controllers would only apply to single input single output feedback loops. In addition, recent nonlinear control techniques also provide additional improvements by allowing processes to be run over a larger operating range without controller retuning. This paper presents such an application of model-based nonlinear multivariable control to a cement milling circuit.

The schematic layout of a cement milling circuit is shown in Fig. 1. The mill is fed with raw material (feed). After grinding, the material is introduced in a high-efficiency classifier and separated into two classes. The tailings (refused part) are fed back into the mill while the finished product (accepted part) exits the milling circuit. The classification of the material is driven by the rotational speed and by the air flow rate of the classifier.

Traditionally, the application of feedback control techniques to cement milling circuits is limited to monovariable classical proportional integral (PI) control of the tailings flow rate (or of the circulating load) with either the feed flow rate or the classifier speed as control action. A typical example can be found in [1].

Recently, multivariable control techniques (based on the linear quadratic control theory) have been introduced to improve the performances of the milling circuit [2]. However, this controller, whose design is based on a linear approximation of the process, is only effective in a limited range around the nominal operating conditions. On some occasions, it has been observed on real plants that important changes in operating

Manuscript received April 16, 1997; revised May 2, 1998. This work was supported in part by the "S.A. Slegten" Company, Belgium and by MURST project "Model Identification, System Control, Signal Processing."

L. Magni is with the Dipartimento di Informatica e Sistemistica, Università di Pavia, 27100 Pavia, Italy.

G. Bastin and V. Wertz are with Université Catholique de Louvain, CESAME, Bât. Euler, 1348 Louvain-la-Neuve, Belgium.

Publisher Item Identifier S 1063-6536(99)05857-1.

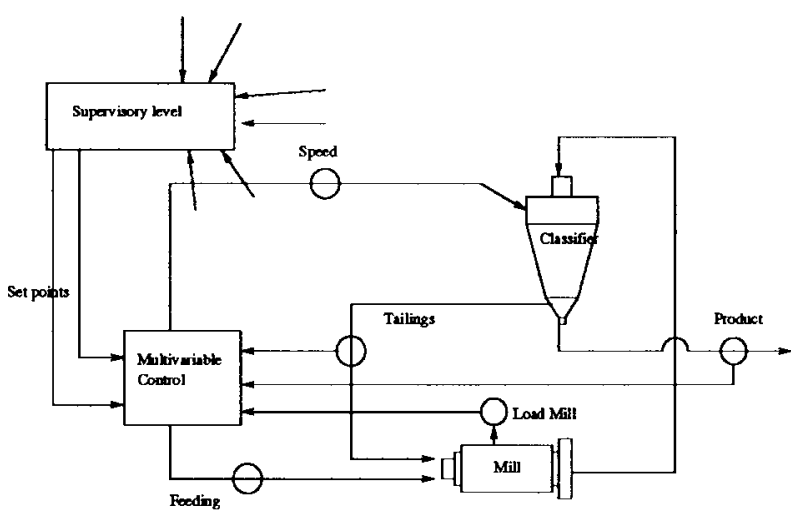

Fig. 1. Milling circuit principle.

conditions (due for example to changes of the hardness of the clinker) have driven the mill to a region where the controller cannot stabilize the plant.

This paper presents a nonlinear model of a cement mill motivated by practical observations about the linear quadratic Gaussian (LQG) controller on an industrial milling circuit [3]. Starting from this nonlinear model a multivariable nonlinear receding horizon (NRH) control strategy based on the algorithm presented in [5]-[7] is applied to improve the performance and to enlarge the stability region obtained with the LQ controller that is now in operation. With this strategy, two outputs are simultaneously controlled (finished product and load in the mill) by using the two available inputs (feed and classifier speed).

This paper is organized as follows. The control strategy is described in Section II. Then, the nonlinear model required to take into account the changes of hardness of input material is described in Section III. The next section describes an LQ controller designed on the basis of this nonlinear model. This controller is similar (although not exactly identical) to the LQ controller that has been described in [2], and exhibits similar instabilities as those that have been occasionally observed on real plants. Section V describes the nonlinear predictive controller which has been designed and the simulation results which have been obtained.

\section{Control Strategy}

In steady-state operation, it is clear that the product flow rate is necessarily equal to the feed flow rate while the tailings flow rate and the load in the mill may take any arbitrary constant values. The load in the mill depends on the input 
feed (fresh feed plus tailings flow rate) and on the output flow rate that depends in a nonlinear way on the load in the mill and on a very important and time-varying quantity: the hardness of the material. Sometimes this nonlinearity can also cause the instability of the system and the obstruction of the mill (a phenomenon called "plugging"), which then requires an interruption of the grinding process. These considerations motivated the introduction of a nonlinear model of the plant [4]. The load in the mill must be controlled at a well chosen level because too high a level of the load in the mill leads to the obstruction of the mill, while too low a circulating load contributes to fast wear of the mill internal equipment. Moreover, the energy consumption of the mill (i.e., the ratio energy per unit product) depends on the output of the mill that is related to the load in the mill. A usual approach is to control the tailings flow rate by using the feed flow rate as control input. This control strategy is, however, not fully satisfactory since it indirectly induces a loss of control of the product flow rate.

A correct fineness of the product is also very important [2]. The fineness depends on the composition of the mill feed, but also on the rotational speed and on the air flow rate of the classifier. A natural control objective would therefore be to keep the fineness as close as possible to a desired value by acting, for example, on the rotational speed of the classifier. However, in the usual case where on-line fineness measurements are not available, an indirect control strategy is necessary.

The following can also be observed in practice.

- For a given feed composition, the fineness will be constant if the product flow rate and the load in the mill are kept constant.

- Similarly the energy consumption of the process (ratio energy/unit product) will be fully under control if the product flow rate and the load in the mill are regulated at suitable set points.

This clearly implies that an overall control of both the fineness and the energy consumption can be achieved at a supervisory level in the control system, by manipulating and optimizing the set points of the tailings flow rate or of the product flow rate or of the load in the mill, using for instance off-line laboratory measurements of the fineness.

\section{Nonlinear Continuous-Time Model}

In this section we present the nonlinear model of the milling circuit which has been introduced in [8]. This model should describe the evolution of the load in the mill and it should be able to reproduce some (unstable) behavior that has been observed with the LQ controller in the feedback loop. The model consists of three nonlinear differential equations explaining the evolution of three states

$$
\left\{\begin{aligned}
T_{f} \dot{y}_{f} & =-y_{f}+(1-\alpha(z, v, d)) \varphi(z, d) \\
\dot{z} & =-\varphi(z, d)+u+y_{r} \\
T_{r} \dot{y}_{r} & =-y_{r}+\alpha(z, v, d) \varphi(z, d)
\end{aligned}\right.
$$
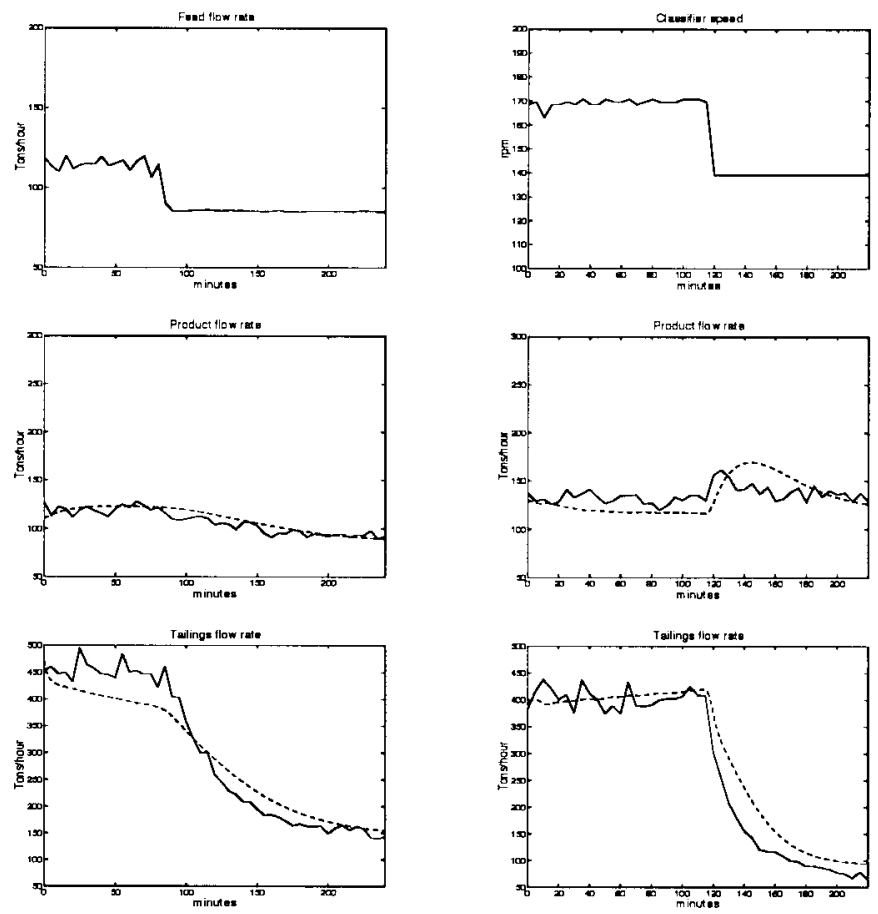

Fig. 2. Comparison of measured (solid line) and modeled (dotted line) step responses.

with

$$
\begin{aligned}
\alpha(z, v, d) & =\frac{\varphi^{m} v^{n}}{K_{\alpha}+\varphi^{m} v^{n}} \\
K_{\alpha} & =(570)^{m} 170^{n}\left(\frac{570}{450}-1\right)(\text { Tons } / \mathrm{h})^{m} \mathrm{r} / \mathrm{min}^{n} \\
m & =0.8 \quad n=4 \\
\varphi(z, d) & =\max \left\{0 ;\left(-d K_{\varphi 1} z^{2}+K_{\varphi 2} z\right)\right\} \\
K_{\varphi 2} & =16.50(\mathrm{~h})^{-1} \quad K_{\varphi 1}=0.1116(\text { Tons * } \mathrm{h})^{-1} \\
T_{f} & =0.3 \mathrm{~h} \quad T_{r}=0.01 \mathrm{~h} \quad d=1
\end{aligned}
$$

where $y_{f}$ is the product flow rate (Tons/h), $z$ is the load in the mill (Tons), $y_{r}$ is the tailings flow rate (Tons $\left./ \mathrm{h}\right), \varphi(z, d)$ is the output flow rate of the mill (Tons/h), $u$ is the feed flow rate (Tons $/ \mathrm{h}), v$ is the classifier speed $(\mathrm{r} / \mathrm{min})$, and $d$ represents the hardness of the material inside the mill with respect to the nominal one. The values of the various coefficients in this nonlinear model have been tuned in such a way that the model step responses fit with experimental step responses described in [3] (see Fig. 2).

From the second nonlinear differential equation and the definition of $\varphi$, it is possible to notice that there is a constraint on the maximum value of the circulating load (feed plus tailings flow rates). By an easy manipulation of the three nonlinear differential equations it follows that it is possible to choose independently only two of the three steady-state values, the third one being imposed from the model equations. Choosing setpoints for $y_{f}$ and $y_{r}$ would impose a given steadystate value for $\varphi$ which could be unachievable. Instead, we suggest here to choose the load in the mill $z$ and the value 
of the product flow rate $y_{f}$ or of the tailings flow rate $y_{r}$ as set-points.

The main problem observed on industrial milling circuits, controlled by the "linear quadratic multivariable controller," is caused by large changes of the feed hardness. In some cases, the LQ controller is not able to control the mill after such large changes, plugging occurs in the mill and an interruption of the grinding process is necessary. So, our aim with the nonlinear model is to reproduce this behavior at least qualitatively, when an LQ controller is applied. To see that the nonlinear model presented here is able to reproduce this practical observation, we will consider first an LQ controller based on the linearized approximation of the nonlinear model and we will show that simulations in closed-loop with the nonlinear model do exhibit instability when the hardness changes. Then, a nonlinear control law will be introduced to overcome this problem without loosing the good nominal performance of the LQ controller.

\section{LQ Control Design}

The nonlinear model (1) is first expanded with the following two sets of equations in order to ensure zero steady-state error and weighting of the control increments as well as of the control variables:

$$
\begin{aligned}
& \left\{\begin{array}{l}
\dot{u}=d u \\
\dot{v}=d v
\end{array}\right. \\
& \left\{\begin{array}{l}
\dot{\sigma}_{y f}=y_{f}^{*}-y_{f} \\
\dot{\sigma}_{z}=z^{*}-z .
\end{array}\right.
\end{aligned}
$$

With the model (1)-(3), zero steady-state error will be achieved provided asymptotic stability is obtained with the feedback controller. Note that parameter changes may modify the equilibrium values for some of the states, but (3) guarantees zero steady-state error for the two selected outputs.

Let us rewrite the system (1)-(3) as

$$
\dot{x}=f_{c}(x, U)
$$

with

$$
x=\left[\begin{array}{c}
y_{f} \\
z \\
y_{r} \\
u \\
v \\
\sigma_{y f} \\
\sigma_{z}
\end{array}\right] \quad U=\left[\begin{array}{l}
d u \\
d v
\end{array}\right] \quad x^{*}=\left[\begin{array}{c}
y_{f}^{*} \\
z^{*} \\
y_{r}^{*} \\
u^{*} \\
v^{*} \\
0 \\
0
\end{array}\right]
$$

where $x^{*}$ is such that

$$
0=f_{c}\left(x^{*}, 0\right)
$$

In order to investigate some properties of the linearized system, define

$$
A\left(x^{*}\right)=\left.\frac{\partial f_{c}(x, U)}{\partial x}\right|_{x=x^{*}, U=0} \quad B=\left.\frac{\partial f_{c}(x, U)}{\partial U}\right|_{x=x^{*}, U=0}
$$

Then the system

$$
\dot{x}_{L}=A\left(x^{*}\right) x_{L}+B U
$$

with $x_{L}=x-x^{*}$ is the linearized system corresponding to system (4) around the equilibrium point $(x, U)=\left(x^{*}, 0\right)$. Note that the linearized system depends only on the values of $v^{*}$ and $z^{*}$.

The corresponding state-space representation in discrete time is given by

$$
x_{L}(k+1)=A_{D}\left(x^{*}\right) x_{L}(k)+B_{D}\left(x^{*}\right) U(k)
$$

where $k$ is an integer index and $A_{D}\left(x^{*}\right)$ and $B_{D}\left(x^{*}\right)$ are obtained in a standard way from $A\left(x^{*}\right)$ and $B$ with a sampling period $T_{e}=1 \mathrm{~min}$. The LQ-controller, based on the discrete-time linear system state-space representation, has been designed using the Matlab Control System Toolbox. Recall that the design is based on the computation of the matrix gain $K\left(x^{*}\right)$ of the linear controller

$$
U(k)=-K\left(x^{*}\right) x_{L}(k)
$$

which minimizes the quadratic criterion

$$
J\left(x^{*}\right)=\sum_{i=0}^{\infty} x_{L}(t+i)^{\prime} Q x_{L}(t+i)+U^{\prime}(t+i) R U(t+i)
$$

subject to (6), where $Q$ and $R$ are two symmetric positive definite matrices.

In Figs. 3 and 4 we present two simulations obtained with $Q=I$ and $R=I * 10^{-3}$. This choice is similar to the one taken in [2], which has been applied on several industrial cement grinding circuits. Starting from a general equilibrium point $x^{*}=\left[\begin{array}{lllll}120 & 55450120170 & 0 & 0\end{array}\right]^{\prime}$, we choose the setpoint $\left[\begin{array}{ll}y_{f}^{*} & z^{*}\end{array}\right]^{\prime}=\left[\begin{array}{lll}120 & 75\end{array}\right]^{\prime}$, corresponding to the maximum of the $\varphi$ curve which is the optimum from an economical point of view, and then, we suppose that the hardness $d$ changes from its nominal value 1 to 1.25 in the first example (Fig. 3) and from its nominal value 1 to 1.34 in the second example (Fig. 4). For the $L Q$ controller (dotted lines) it is clear that the nominal response to a set-point change in $z$ is correct but that the rejection of a hardness change is poor in the first case while in the second case this perturbation causes instability of the closed loop (plugging).

These simulations show that the nonlinear model is able to represent the nonlinear behavior of the plant when the hardness changes. Hence the nonlinear model can be considered as a good model to synthesize a control law whose aim is to control the cement mill in a larger operating range.

\section{Predictive Control LaW}

In this section the nonlinear receding horizon (NRH) control law, first presented in [5] and then extended in [6] and [7], is used to control the cement mill. This NRH control technique uses a suitable nonquadratic terminal state penalty retaining the computational advantages of finite-horizon optimization 

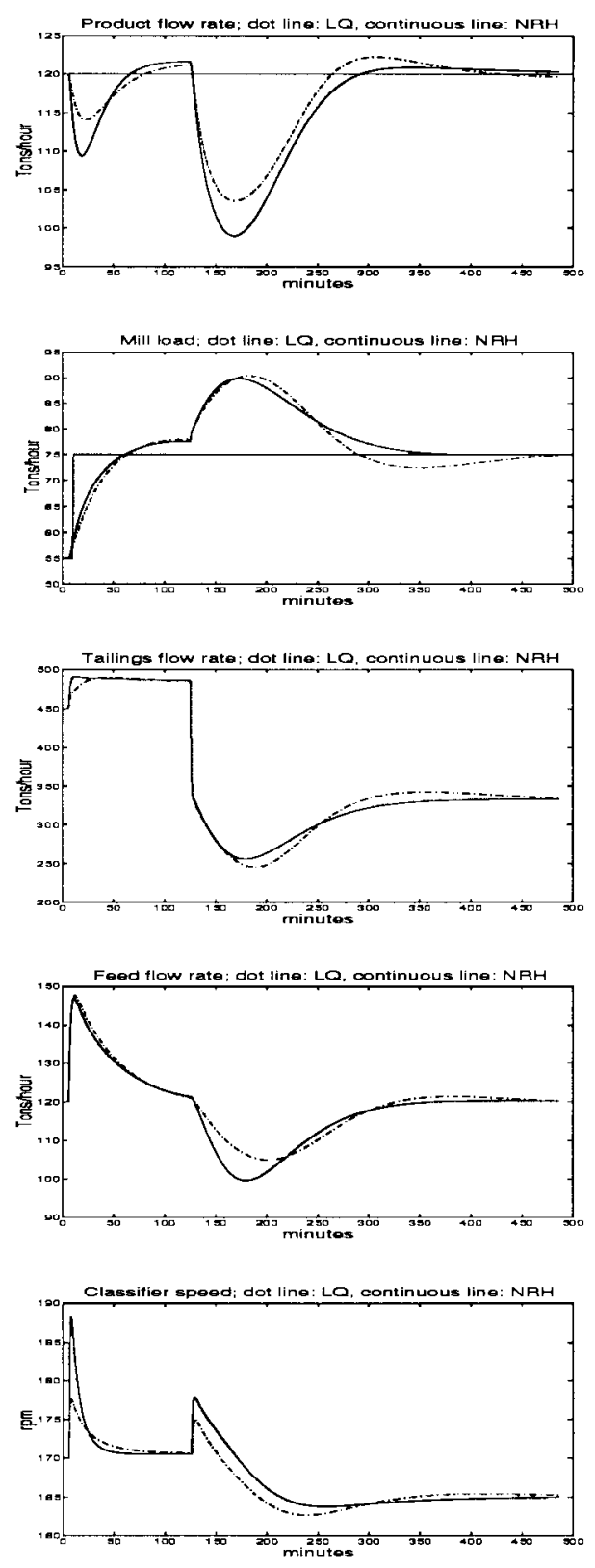

Fig. 3. Step in $z^{*}$ from 55 to 75 after 6 min and in $d$ from 1 to 1.25 after $126 \mathrm{~min}$.

without terminal constraints and ensuring the closed loop stability under very mild conditions (only the stabilizability of the linearized system is required).

It will be clear in the sequel that the NRH regulator proposed here can be seen as a consistent nonlinear extension of the local linear control law. This observation justifies the use of this control law to improve the performance of an LQ controller when there are constraints or nonlinearities. The application of the NRH control law to the present problem goes as follows.

Consider the nonlinear discrete-time system

$$
x(k+1)=f(x(k), U(k)), x(t)=\bar{x}, k \geq t
$$

obtained from system (4) by discretization holding the manipulated variables $U$ constant over the sampling interval. The
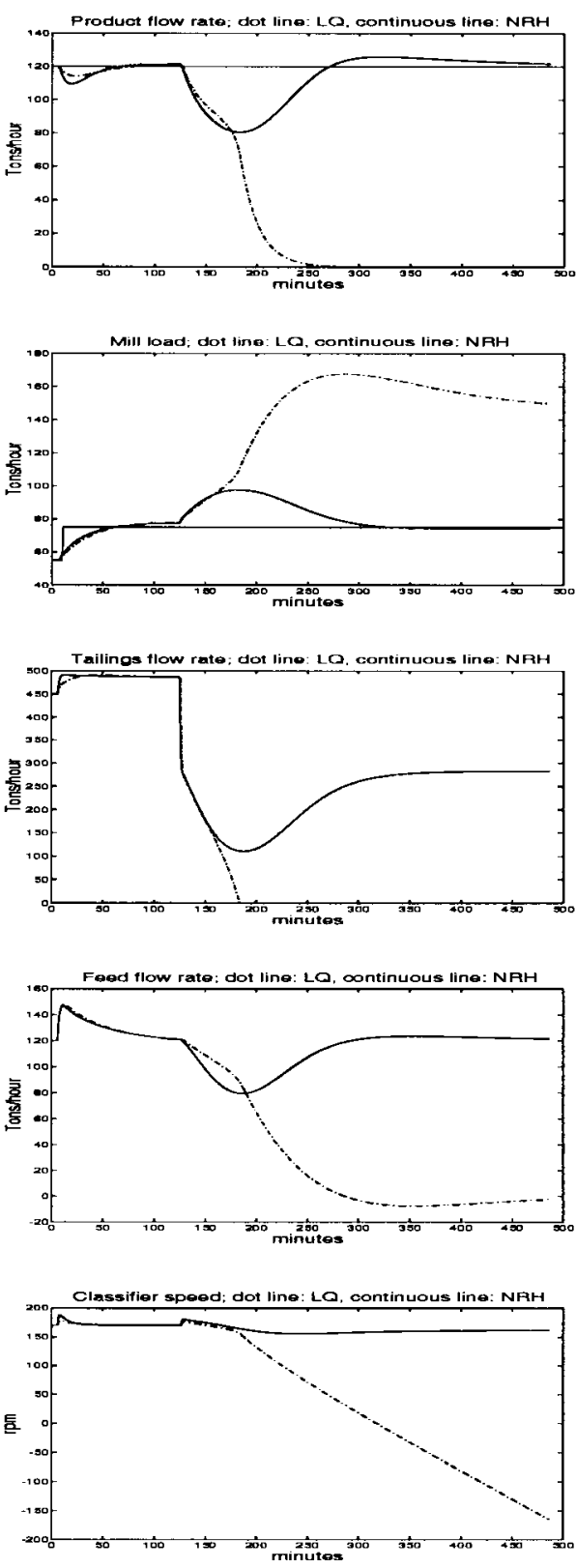

Fig. 4. Step in $z^{*}$ from 55 to 75 after 6 min and in $d$ from 1 to 1.34 after $126 \mathrm{~min}$.

state and the input vectors are subject to the constraints

$$
x(k) \in \mathcal{X}, U(k) \in \mathcal{U}, k \geq t
$$

where $\mathcal{X}$ and $\mathcal{U}$ are closed and bounded subsets of $R^{7}$ and $R^{2}$, respectively, containing $x^{*}$ and the origin, respectively, as an interior point.

Define now the set:

$X\left(K\left(x^{*}\right)\right)=\left\{\bar{x} \in R^{7} \mid \lim _{k \rightarrow \infty}\|x(k)\|=x^{*}\right.$, subject to (9) with $\left.x(t)=\bar{x}, x(k) \in \mathcal{X}, U(k)=K\left(x^{*}\right) x(k) \in \mathcal{U}, k \geq t\right\}$.

In other words, $\bar{x}$ belongs to $X\left(K\left(x^{*}\right)\right)$ if the application of the LQ control law (7) to the nonlinear system (9) generates an input sequence guaranteeing the satisfaction of the constraints (10) and driving the state to the equilibrium point 
$x^{*}$ asymptotically. Then consider the following finite-horizon problem.

\section{Finite-Horizon Optimal Control Problem (FHOCP)}

Minimize, with respect to $U_{t, t+N-1}:=[U(t) U(t+1) \ldots U(t+$ $N-1)]$ the cost function

$$
\begin{aligned}
& J\left(\bar{x}, U_{t, t+N-1}, N, x^{*}\right) \\
& =\sum_{i=0}^{N-1}\left\{\left(x(t+i)-x^{*}\right)^{\prime} Q\left(x(t+i)-x^{*}\right)\right. \\
& \left.\quad+U^{\prime}(t+i) R U(t+i)\right\}+V_{f}\left(x(t+N), x^{*}\right)
\end{aligned}
$$

subject to (9) with $Q$ and $R$ defined above. In (11) the nonquadratic terminal state penalty is defined as

$$
\begin{aligned}
& V_{f}\left(x(t+N), x^{*}\right) \\
& =\sum_{i=N}^{\infty}\left\{\left(x(t+i)-x^{*}\right)^{\prime} Q\left(x(t+i)-x^{*}\right)\right. \\
& \left.\quad+\left(x(t+i)-x^{*}\right)^{\prime} K\left(x^{*}\right)^{\prime} R K\left(x^{*}\right)\left(x(t+i)-x^{*}\right)\right\}
\end{aligned}
$$

In other words, $V_{f}\left(x(t+N), x^{*}\right)$ is the cost that is incurred over $[t+N, \infty]$ by applying the LQ control law (7) to the nonlinear system (9). Note that $V_{f}\left(x(t+N), x^{*}\right)$ is finite if $x(t+N)$ can be driven to $x^{*}$ by the linear state feedback controller. In the following, given $K\left(x^{*}\right)$ such that $A_{D}\left(x^{*}\right)+B_{D}\left(x^{*}\right) K\left(x^{*}\right)$ is stable, a sequence $U_{t, t+N-1}$ is said admissible if when applied to (9) the following constraints are satisfied.

C1: $x(k) \in \mathcal{X}, u(k) \in \mathcal{U}, k=t, \cdots, t+N-1$, where $\mathcal{X}$ and $\mathcal{U}$ represent state and control constraint sets and are closed and bounded subsets of $R^{7}$ and $R^{2}$ containing $x^{*}$ and the origin, respectively, as an interior point.

C2: $x(t+N) \in X\left(K\left(x^{*}\right)\right)$ where $X\left(K\left(x^{*}\right)\right)$ is the set of states such that the application of the LQ control law (7) to the nonlinear system (9) starting from these states generates an input sequence guaranteeing the satisfaction of the above constraints and driving the state to the equilibrium point $x^{*}$ asymptotically.

Associated with (11), the following predictive NRH control strategy is introduced.

\section{Predictive Control Law}

Given the LQ time invariant control law, $K\left(x^{*}\right)$, define $\bar{x}=x(t)$ and find an admissible control sequence $U_{t, t+N-1}^{o}$ solving the FHOCP. Then, apply the control $U(t)=U^{o}(\bar{x})$, where $U^{o}(\bar{x})$ is the first column of the optimal sequence $U_{t, t+N-1}^{o}$ and solve the same problem again at time $t+1$ (receding horizon strategy).

In the following, $X_{0}\left(N, x^{*}\right)$ will denote the set of states $\bar{x}$ such that the NRH control $U^{o}(\bar{x})$ is computable (i.e., the set of admissible sequences is nonempty). The stabilizing property of the predictive NRH controller is then stated in the following theorem.
Theorem [5]: Assume that $A_{D}\left(x^{*}\right)+B_{D}\left(x^{*}\right) K\left(x^{*}\right)$ is stable. Then, if the NRH control law $U=U^{\circ}(x)$ is applied to the nonlinear system (9), the equilibrium point $x^{*}$ is an asymptotically stable equilibrium point of the resulting closedloop system with region of attraction $X_{0}\left(N, x^{*}\right)$.

Remark 1: Note that in view of the enlargement of the system with the integral action on the tracking error (3), the NRH control law guarantees zero steady-state error for the perturbed system if asymptotic stability is achieved.

Remark 2: As seen from the statement of the theorem, the choice of the optimization horizon $N$ is immaterial as far as local stability is concerned. However, it is clear that increasing $N$ is likely to enlarge the domain of attraction of the equilibrium point. This is an argument in favor of long optimization horizons, although computational constraints could pose a practical upper limit (the difficulty of solving the FHOCP obviously increases with $N$ ).

The computation of $V_{f}\left(x(t+N), x^{*}\right)$ in (12) is performed by iterating

$$
x(k+1)=f\left(x(k), K\left(x^{*}\right) x(k)\right), \quad k \geq t+N .
$$

In theory, this iteration should be performed for an infinite number of time steps. In practical a good idea is to approximate the terminal penalty $V_{f}\left(x(t+N), x^{*}\right)$ in (12) as

$$
\begin{aligned}
& V_{f}\left(x(t+N), x^{*}\right) \\
& \cong \sum_{i=N}^{N+M-1}\left\{\left(x(t+i)-x^{*}\right)^{\prime} Q\left(x(t+i)-x^{*}\right)\right. \\
& \left.\quad+\left(x(t+i)-x^{*}\right)^{\prime} K\left(x^{*}\right)^{\prime} R K\left(x^{*}\right)\left(x(t+i)-x^{*}\right)\right\} \\
& \quad+\left(x(t+N+M)-x^{*}\right)^{\prime} P\left(x^{*}\right)\left(x(t+N+M)-x^{*}\right)
\end{aligned}
$$

where $P\left(x^{*}\right)$ is the unique nonnegative solution of the algebraic Riccati equation associated with the solution of the LQ control law and $M$ is such that $M+N$ is "large" compared to the system dynamics. The rationale behind this choice lies in the assumption that at time $t+N+M$ the system has been driven in a sufficiently small neighborhood of the equilibrium point $x^{*}$ by the application of the sequence $U_{t, t+N-1}^{o}$ followed by the linear control law $U(t+N+j)=$ $K\left(x^{*}\right) x(t+N+j), j=0, \cdots, M-1$. In this neighborhood, the behavior of the system is approximately linear, so that, by standard results of LQ control theory

$$
\begin{aligned}
& \sum_{k=N+M}^{\infty}\left\{\left(x(t+i)-x^{*}\right)^{\prime} Q\left(x(t+i)-x^{*}\right)\right. \\
& \left.+\left(x(t+i)-x^{*}\right)^{\prime} K\left(x^{*}\right)^{\prime} R K\left(x^{*}\right)\left(x(t+i)-x^{*}\right)\right\} \\
& \quad \cong\left(x(t+N+M)-x^{*}\right)^{\prime} P\left(x^{*}\right)\left(x(t+N+M)-x^{*}\right) .
\end{aligned}
$$

Thanks to the above considerations the computational burden is significantly reduced. In the present application the FHOCP is solved using the Matlab Optimization Toolbox without any computational problems.

As for the tuning of the state and input weighting matrices $Q$ and $R$, observe that the nonlinear control law $U=\gamma^{\mathrm{NRH}}(x)$ can be seen as a consistent extension of $U=K\left(x^{*}\right) x$, in the 


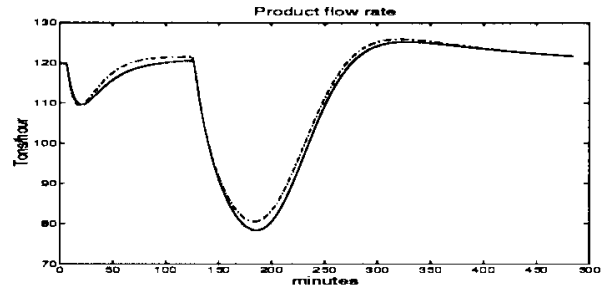

Fig. 5. Control law synthesis with linear $\alpha$ (solid line) and with nonlinear $\alpha(z, v, d)$ (dotted line).

sense that

$$
\left.\frac{\partial \gamma^{\mathrm{NRH}}(x)}{\partial x}\right|_{x=x^{*}}=K\left(x^{*}\right) .
$$

Consequently, it is reasonable to tune $Q$ and $R$ so as to optimize the closed-loop performance of the linearized system trusting that the nonlinear $\mathrm{RH}$ controller will be able to guarantee a satisfactory performance in a larger domain.

\section{A. Results}

Consider now the application of this nonlinear predictive control strategy to the cement mill model and its comparison with a classical LQ controller based on the linear approximation of the model. In Figs. 3 to 4 the two simulations with the LQ controller are compared with the ones obtained using the NRH control law with the same $Q$ and $R$ and with $N=2$ (continuous line). Note that the nominal performance, when the set-point in $z$ changes, is very similar to the one obtained with the LQ controller. Similar conclusions can also be drawn when the hardness changes, as long as the LQ controller is still able to stabilize the plant. In that case (see Fig. 3), the performances of both controllers are quite comparable. However, in Fig. 4 it is clear that the NRH control stabilizes the plant even when the LQ controller can not.

These simulations show that if the nonlinear model is a good representation of the nonlinear behavior of the plant in the case of hardness changes inside the mill, then the nonlinear $\mathrm{RH}$ is able to solve one problem experienced with the LQ controller that is now in operation.

Remark 3: One of the main problems of model-based algorithms is that it is not always possible to have a precise knowledge of all parameters of the model of the process to be controlled. Hence, it is important for the control algorithm to be robust with respect to some modeling uncertainties. As an indication of such robustness for the present algorithm, we have performed the following simulation. The process model is still the same nonlinear model (1) but the controller is computed on the basis of a simplified version where $\alpha(z, v, d)=\alpha_{1}+\alpha_{2} v$. In Fig. 5 we report this simulation under the same conditions as in Fig. 4. It is clear that the simplified model used in the computation of the control law does not alter the performance of the controller.

Remark 4: In our simulations we have chosen a sampling period $T_{e}=1 \mathrm{~min}$ and a control horizon $N=2$. In some real plants the LQ controller operates with a sampling period $T_{e}=5 \mathrm{~min}$. In this case, using the same control horizon $N=2$ and hence with the same computational burden, the

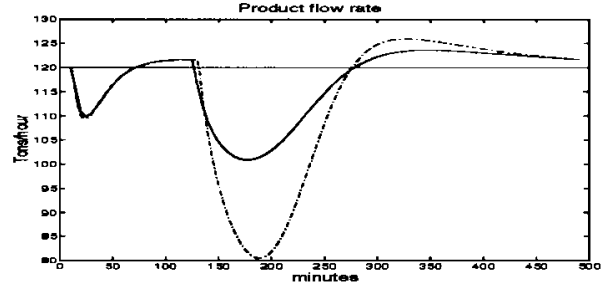

Fig. 6. Comparison between $T_{e}=5 \mathrm{~min}$ (solid line) and $T_{e}=1 \mathrm{~min}$ (dotted line).

NRH control law achieves a better performance because with a longer sampling time the FHOCP is based on a longer behavior of the plant. In Fig. 6 the simulation example of Fig. 4 with sampling period $T_{e}=5 \mathrm{~min}$ is compared with the one with $T_{e}=1 \mathrm{~min}$.

\section{DISCUSSION}

The aim of this work has been to investigate further improvements to the multivariable LQ control law that was recently developed and succesfully implemented on several cement milling circuits. In particular practical observations had shown the appearance of mill plugging on some occasions, a phenomenon which the $L Q$ controller could not prevent from happening. In this paper, we have presented a nonlinear model which specifically models the load in the mill, and subsequently a nonlinear controller which preserves the main characteristics of the previous LQ controller but enlarges the operating region in which plugging can be avoided. (See also [8], where a controller with global stabilizing properties is presented.)

The real difficulty of the proposed controller is the need for a measurement of the load of the mill. Various techniques can be implemented (some of them already exist on industrial milling circuits).

- The mill itself could be installed on a weighing device so that its total mass can be measured and the cement load deduced from it.

- On some mills, an "electronic ear" measures the noise of the mill, which is inversely proportional to the load in the mill.

Contrary to general intuition, the electrical power of the mill motor cannot be used as a indirect measurement of the load in the mill. Indeed, it can be shown that the mill power is first increasing with load, reaching an maximum value which is actually the optimal operating point, and then decreasing when the load increases further. If precise measurements of the load cannot be obtained, then a nonlinear observer of this variable should be studied as an alternative to the presented scheme. This is left to future work.

\section{ACKNOWLEDGMENT}

This paper presents research results of the Belgiam Program on Interuniversity Attraction Poles, initiated by the Belgiam State, Prime Minister's Office for Science, Technology and Culture. The scientific responsibility rests with its authors. 
The multivariable control structure mentioned in the paper is protected by a patent application (Patent No. 93870 040.8).

\section{REFERENCES}

[1] C. Ciganek and K. Kreysa, "Two-parameter control system for a cement grinding process," Translation of Zement-Kalk-Gips, pp. 202-206, 1991.

[2] V. Van Breusegen, L. Chen, G. Bastin, V. Wertz, V. Werbrouck, and C. de Pierpont, "An industrial application of multivariable linear quadratic control to a cement mill circuit," IEEE Trans. Ind. Applicat., vol. 32, pp. 670-677, 1996.

[3] V. Van Breusegen, L. Chen, V. Werbrouck, G. Bastin, and V. Wertz, "Multivariable linear quadratic control of a cement mill: An industrial application," Contr. Eng. Practice, vol. 2, pp. 605-611, 1994.
[4] B. de Haas, F. Jadot, G. Bastin, and V. Wertz, CESAME, Int. Res. Rep., 1996.

[5] G. De Nicolao, L. Magni, and R. Scattolini, "Stabilizing nonlinear receding horizon control via a nonquadratic terminal penalty," in Proc. IMACS Multiconf. CESA'96, Lille, France, vol. 1, pp. 185-187, July 1996.

[6] G. De Nicolao, L. Magni, and R. Scattolini, "Stabilizing receding horizon control of nonlinear time-varying systems," IEEE Trans. Automat. Contr., vol. 43, pp. 1030-1036, 1998.

[7] G. De Nicolao, L. Magni, and R. Scattolini, "Stabilizing predictive control of NARX models," Automatica, vol. 33, pp. 1691-1698, 1997.

[8] F. Jadot, G. Bastin, V. Wertz, and L. Magni, "Preventing cement mills from plugging by robust state feedback," in Proc. Contr. 97, Sydney, Australia, Oct. 1997, pp. 248-251. 